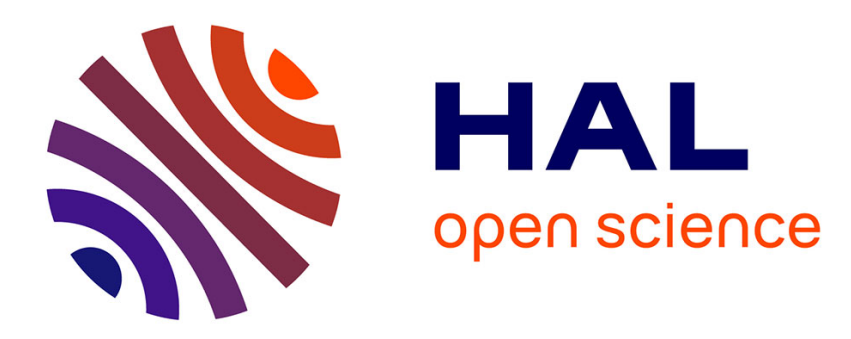

\title{
Étude du catalogage et des inventaires de la bibliothèque musicale de l'ancien Concert de Lyon
}

Bénédicte Hertz

\section{To cite this version:}

Bénédicte Hertz. Étude du catalogage et des inventaires de la bibliothèque musicale de l'ancien Concert de Lyon. Dix-Huitième Siècle, 2011, Le monde sonore, 43, pp.143-163. 10.3917/dhs.043.0143 . hal02888908

\section{HAL Id: hal-02888908 https://hal.science/hal-02888908}

Submitted on 3 Jul 2020

HAL is a multi-disciplinary open access archive for the deposit and dissemination of scientific research documents, whether they are published or not. The documents may come from teaching and research institutions in France or abroad, or from public or private research centers.
L'archive ouverte pluridisciplinaire HAL, est destinée au dépôt et à la diffusion de documents scientifiques de niveau recherche, publiés ou non, émanant des établissements d'enseignement et de recherche français ou étrangers, des laboratoires publics ou privés. 


\section{Étude du catalogage et des inventaires de la bibliothèque musicale de l'ancien Concert de Lyon ${ }^{1}$ (Bénédicte Hertz)}

$\mathrm{Au}$ siècle des Lumières, les bibliothèques se multiplient dans la ville de Lyon, conséquence directe de l'essor de l'édition. La cité en compte plusieurs, se distinguant les unes des autres par leur taille, leur spécificité ou encore la rareté des ouvrages qu'elles rassemblent. Parallèlement aux collections exclusivement privées, s'ouvrent, sur l'exemple parisien, de petits cabinets de lecture ou des bibliothèques semi-publiques, qui appartiennent souvent à des religieux. Ainsi en est-il des Jésuites, au grand collège de la Trinité et à la maison Saint-Joseph, ou des Augustins de Saint-Vincent, ces derniers ayant récupéré la bibliothèque de Gacon, un ancien échevin de la ville. Car la plupart des fonds sont constitués des collections particulières de Lyonnais érudits : l'abbé La Croix, Ruffier d'Attigna ou Clapeyron, trésoriers de France, Dugas et La Tourette de Fleurieu, anciens prévôts, Michon, avocat du roi, La Valette, conseiller honoraire de la cour des monnaies, etc ${ }^{2}$. Vers 1750, la seule bibliothèque véritablement publique de la cité est l'ancienne collection particulière de Pierre Aubert, léguée de son vivant au consulat de Lyon, en 1731. Elle devint accessible aux lettrés qui le désiraient en 1733, à la suite du décès de son propriétaire. Le consulat n'ouvre au public la célèbre collection des Jésuites qu'en 1765, trois ans après leur départ du collège de la Trinité, et l'adjoint alors à celle de Pierre Aubert. Les autres bibliothèques, bien que

\footnotetext{
${ }^{1}$ Ce texte doit beaucoup aux conseils et relectures attentives de Thomas Vernet et Christophe Schmit. Qu'ils en soient ici remerciés.

${ }^{2}$ Sur toutes ces personnalités, voir: Patrice Béghain et coll. éd., Dictionnaire historique de Lyon, Lyon, Stéphane Bachès, 2009, 1504 p.
} 
privées, sont pourtant notoires ; à l'instar de celle de l'académie des sciences, belles-lettres et arts, elles sont recensées annuellement dans l'Almanach local.

La musique profite de l'immense appétit de savoir général qui caractérise cette période. De nombreux amateurs collectionnent traités, partitions et recueils, dont le raffinement de la copie est parfois remarquable. Lyon sera privilégiée parmi toutes les villes de province de l'Ancien régime: son Concert va se doter d'une collection de musique qui passera en son temps pour une des plus riches et des plus belles du royaume, ce qu'attestent grandement les sources manuscrites et imprimées conservées aujourd'hui à la bibliothèque municipale ${ }^{3}$. Le Concert est fondé en 1713 par Jean-Pierre Christin et Nicolas-Antoine Bergiron de Briou du Fort-Michon, sous le titre d' " académie des beaux-arts ». Il se confond à ses débuts avec l'académie des sciences et belles-lettres lyonnaise, créée en 1700. L'académie des beaux-arts, sous la protection du maréchal-duc de Villeroy, est officialisée en 1724 par des lettres patentes et des statuts $^{4}$. Ceux-ci prévoient une séance musicale hebdomadaire, offrant à ses académiciens un programme varié et du meilleur goût : motets à grand chœur, fragments d'œuvre lyrique côtoient, plus en avant dans le siècle, cantatilles, ariettes, motets à voix seules et pièces instrumentales. Cette même année, alors que les sociétaires amateurs laissent progressivement la place, dans l'orchestre et le chœur, à des musiciens professionnels, l'académie fait construire sa propre salle de concert, sur la place des Cordeliers. Cet «hôtel du Concert » accueillera la bibliothèque musicale dans une de ses pièces qui lui sera particulièrement dédiée. Le Concert tient aussi, dès 1736, des réunions d'échange d'idées et de conversations sur la musique, part de son activité qui sera rattachée en 1758 à l'académie scientifique, la nouvelle institution devenant alors l'«académie des sciences, belles-lettres et arts », dénomination qui perdura. En 1741, le consulat de Lyon, pour sauver son Concert de la faillite, rachète ses biens mobiliers et immobiliers, tout en lui en laissant le total usufruit. Malgré de nombreuses vicissitudes financières, l'académie des beaux-arts se maintient jusqu'en $1773^{5}$. Cette longévité et la constance de son activité inégalées dans le paysage de l'académisme musical français - caractérisent fondamentalement l'histoire et le rayonnement du Concert lyonnais, histoire et rayonnement

\footnotetext{
${ }^{3}$ L'étude de ces sources - et plus spécifiquement celles du motet à grand chœur, soliste et orchestre - est l'objet de notre thèse : Le Grand motet dans les pratiques musicales (1713-1773). Étude des partitions et du matériel conservés à la bibliothèque municipale de Lyon, thèse de doctorat, Université Lumière-Lyon 2, 2010, 2 vol., 522 p. ; 104 p.

${ }^{4}$ Statuts et reglements de l'Academie des Beaux-Arts établie à Lyon par Lettres patentes du Roy, Lyon, André Laurens, 1724, Bibliothèque municipale de Lyon, Fonds Coste, 115 954. On y trouve les lettres patentes, p. 3-6.

${ }^{5}$ La disparition de l'académie des beaux-arts est attestée en 1774.
} 
dont la constitution d'un catalogue et l'étude des inventaires du fonds musical, deux aspects que nous présenterons ici, donnent un aperçu.

La bibliothèque du Concert, constituée exclusivement de livres de musique, est réservée dans ses débuts à l'usage des académiciens. C'est, avant d'être une collection d'apparat, un fonds nécessaire aux concerts hebdomadaires de l'académie, un répertoire rassemblé pour être joué. Presque toutes les partitions sont exécutables, puisqu'elles comportent très souvent des parties de remplissage - parties d'altos instrumentales absentes des partitions réduites - et que leur est associé un jeu de parties séparées, c'est-à-dire un matériel orchestral et vocal. C'est dire que cette bibliothèque a une vocation utilitaire avant tout. C'est en cela qu'elle constitue un témoignage de premier ordre : elle reflète, outre le bon goût de l'époque, une pratique musicale propre à une ville de province, située sur l'axe rhodanien, passage obligé entre la capitale et l'Italie ou la Provence. Si la bibliothèque du Concert est évoquée, dès 1747, dans la rubrique de l'Almanach concernant l'académie des beaux-arts, elle va être mentionnée indépendamment au côté des bibliothèques de la ville de Lyon, à partir de $1753^{6}$. Il est fort possible qu'une relative ouverture de la collection au public ait été d'usage, de façon non-officielle, bien avant cette date. Il est bien précisé dans l'Almanach lyonnais que ces collections sont en libre accès et que l'on y accueille les étrangers avec un soin tout particulier. La mention du nom et de l'adresse du garde de la bibliothèque de l'académie donne d'ailleurs les informations nécessaires au lecteur. La collection ne cessera d'être répertoriée dans la presse lyonnaise, même après la dissolution du Concert.

Cette annonce, que publie chaque année, au $18^{\mathrm{e}}$ siècle, l'Almanach de Lyon, est sans aucun doute la meilleure description que l'on puisse faire de la collection : « La bibliothèque de musique du Concert peut passer pour la plus belle et la plus précieuse du royaume. Elle est composée d'une très grande quantité de motets à grand chœur, des meilleurs auteurs, tels que Lalande, Bernier, Campra, etc. recueillis avec soin ; il y en a aussi qui ont été composés par les meilleurs maîtres d'Italie. Un très grand nombre d'opéras, dont tous ceux de Lulli, qui y sont complets, et d'autres de bons auteurs, avec plusieurs divertissements, y forment un assemblage intéressant de pièces françaises à grand chœur. On y trouve encore des concerto à grande symphonie. Outre ces pièces qui servent à remplir les concerts généraux, cette bibliothèque en renferme beaucoup d'autres qui sont propres aux concerts particuliers, telles

\footnotetext{
${ }^{6}$ Un article paraît pour la première fois en 1747, dans l'Almanach astronomique et historique de la ville de Lyon (Lyon, Aimé Delaroche), à la rubrique « Bibliothèque du Concert ».
} 
que des livres de cantates, sonates à violon seul, duo et trio; et des motets dans tous les genres à une, deux, trois et quatre voix, avec et sans symphonie ; même des oratorio en latin et en italien, genre de musique particulièrement en usage en Italie $^{7} \gg$. Cette bibliothèque est étonnamment riche. On ne peut que s'émerveiller devant la beauté de certaines partitions de motet ou d'opéra par exemple, mais aussi s'étonner de l'attention portée à la confection des reliures, en matériaux simples mais d'un style très soigné. Les différentes académies provinciales ne semblent jamais s'être dotées d'une aussi vaste collection de musique. Le consulat de Lyon, dans ses actes officiels, s'enorgueillit par ailleurs de posséder la bibliothèque la plus grande du royaume. Le fonds de la bibliothèque de l'académie de Lyon constitue au demeurant, encore à l'heure actuelle, le plus gros corpus de grands motets ou de fragments d'œuvres lyriques conservé en région.

L'une des richesses de la bibliothèque lyonnaise réside dans sa classification. Extrêmement précise, celle-ci comporte dix-neuf catégories, possédant chacune son propre catalogue ${ }^{8}$. Les archives lyonnaises ayant conservé ces précieuses listes, nous avons une connaissance très détaillée du répertoire du Concert, alors même que seul un tiers des volumes de musique a été sauvegardé. Le classement est une source considérable d'informations, puisqu'il permet notamment de recenser des œuvres perdues, d'authentifier des manuscrits conservés ou encore de dater des partitions les unes par rapport aux autres.

Le système de classification de la bibliothèque de l'académie répartit la musique par genres; il différencie musique italienne et française, vocale et instrumentale, sacrée et profane, considérant aussi les effectifs. Les grands motets composent l'ordre A, tandis que les petits motets sont répartis en sept catégories (D, E, F, G, H, I et L). À l'intérieur de chaque catégorie, les pièces ne sont pas obligatoirement numérotées, ni répertoriées de façon détaillée. Les ordres $\mathrm{A}, \mathrm{B}, \mathrm{C}, \mathrm{M}$ et $\mathrm{O}$ présentent pourtant un inventaire complet et numéroté. La liste suivante est celle de l'Ordre des pièces de Musique, qui sont dans la Bibliothèque du Concert de l'Académie des Beaux Arts de Lyon'. Nous y indiquons le nombre de pièces par

\footnotetext{
7 Ibid.

${ }^{8}$ Léon Vallas a déjà édité le catalogue intégral de cette bibliothèque, avec le détail de chaque pièce. Voir : $L a$ Musique au dix-huitième siècle. La Musique à l'académie, thèse de doctorat, université de Lyon, Lyon, La Revue musicale de Lyon, 1908, t. 1, p. 153-168. La liste publiée par Vallas ne rend toutefois pas compte de certaines particularités des catalogues manuscrits originaux. Le parti pris dans la disposition des ajouts altère, nous semble-t-il, la compréhension de l'histoire du catalogue : la mise en page du manuscrit donne en effet parfois certaines indications précieuses sur la rédaction du catalogue et la chronologie des ajouts.

${ }^{9}$ Manuscrit, s.d., Bibliothèque municipale de Lyon, Rés. FM 134008.
} 
« numéro » uniquement lorsque le détail y figure, et en utilisant le mot « livre » ou «pièce » lorsqu'il n'existe pas de numérotation.

- A. Motets à grand chœur (284 numéros).

- B. Pièces de musique françaises à grand chœur (155 numéros).

- C. Symphonies à grands concerts (37 numéros).

- D. Motets à voix seule (33 pièces).

- E. Motets à voix seule et symphonies (71 pièces).

- F. Motets à deux voix (56 pièces).

- G. Motets à deux voix et symphonies (28 pièces).

- H. Motets à trois voix (89 pièces).

- I. Motets à trois voix et symphonies (33 pièces et 1 livre).

- K. Oratoires latins.

- L. Partitions de motets à une, deux, trois, quatre voix avec et sans symphonies (2 livres).

- M. Cantates françaises, en partition (11 numéros).

- N. Cantates françaises en parties séparées.

- O. Symphonies en duo et trio (51 numéros).

- P. Oratoires en italien (5 œuvres).

- Q. Cantates italiennes en partition (5 livres).

- R. Cantates italiennes et ariettes en parties séparées.

- S. Airs italiens, fragments d'opéra et cantates en parties séparées.

- T. Airs et concert de trompette marine et de bouches par le $S^{r}$. J. B. Prin.

On le voit, la musique sacrée rassemble plus de la moitié des ordres. Les grands motets figurent en tout premier, l'ordre A comportant un nombre de titres largement supérieur aux autres. Cette catégorie est la plus volumineuse : un motet à grand chœur est un livre à lui seul, alors que les motets à voix seules, ou petits motets, sont reliés en recueils ou cahiers pouvant en assembler une dizaine. Les œuvres lyriques dans le style français tiennent la deuxième place. Elles viennent juste après les grands motets dans le classement (ordre B) mais aussi par leur importance. La bibliothèque rassemble plus de cent cinquante opéras, ballets ou tragédies lyriques, ce qui est exceptionnel. En réalité, ce ne sont pas toutes des œuvres dans leur version originale et complète, mais souvent des «fragments modernes » ou des adaptations en « concerts », c'est-à-dire des extraits remaniés. Les œuvres pouvaient alors être jouées à l'académie dans une version de concert plus adaptée. Les Affiches de Lyon, annonçant les programmes du Concert entre 1759 et 1772, rendent bien compte de cette pratique : une même œuvre lyrique peut être exécutée deux ou trois semaines de suite, sous la forme de deux ou trois parties successives. Les petits motets, assez nombreux aussi, proviennent pour la majorité de la bibliothèque d'Antoine Hedelin, un amateur de musique italienne qui fit don de sa collection à l'académie lyonnais dans les années 1720-1730. Le troisième ordre contient la musique instrumentale, tout comme les ordres $\mathrm{O}$ et $\mathrm{T}$. Les musiques française et italienne n'y 
sont pas dissociées ; on y retrouve concertos, duos et trios. L'ordre T a été ajouté par la suite et est plus original : il est constitué d'un mémoire et de partitions pour la trompette marine, envoyés par Jean-Baptiste Prin, virtuose de cet instrument.

Le règlement de l'académie, joint aux différents catalogues, explique de manière très précise la numérotation dans les différents ordres: "Chaque pièce aura son numéro particulier, qui commencera par 1 à chaque ordre, et sera marqué, outre son numéro, de la lettre qui désigne l'ordre dans lequel elle se trouve. Par exemple, le motet Cum invocarem à grand chœur étant de l'ordre A. $\mathrm{n}^{0} 7$, ainsi des autres, il sera marqué sur son portefeuille et sur la partition A. $n^{0} 7$. Quand il n'y aura ni partition ni portefeuille à de certaines pièces, elles seront marquées sur l'enveloppe, ou sur la $1^{\mathrm{e}}$ partie détachée de la pièce. Par exemple la $6^{\mathrm{e}}$ œuvre de Corelli qui est à grand concert étant de l'ordre C, il [sic] sera C. $\mathrm{n}^{\mathrm{o}} 1$ sur le $1^{\text {er }}$ violon ou si l'on veut de même sur chaque couverture des parties. Le motet à voix seule et symphonie In convertendo par $\mathrm{M}^{\mathrm{r}}$ Valette, qui est de l'ordre E, pourra être marqué E. $\mathrm{n}^{\mathrm{o}} 1 .^{10}$ » Ce classement simple et facile d'usage, puisqu'il permet d'ajouter à l'infini des numéros aux ordres, est complété par la règle suivante : "Il faut aussi que ces motets particuliers aient aussi des marques particulières suivant les voix dont ils se trouveront. Par exemple pour les motets à voix seule et symphonie qui sont de l'ordre E, pour ceux qui seront pour les dessus pourront être marqués E. $n^{o}$ tel, ceux pour la h[aute]-contre E.E $\left[n^{\circ}\right]$-, en commençant toujours chaque genre de voix par le $\mathrm{n}^{\mathrm{o}} 1$ et laisser du vide dans le grand inventaire pour $\mathrm{y}$ ajouter les motets de la même espèce qui pourront venir dans la suite à l'académie, ceux pour la taille E.E.E. $\mathrm{n}^{\mathrm{o}}$ - et ceux pour la basse E.E.E.E. $\mathrm{n}^{\mathrm{o}}-{ }^{11}{ }^{\gg}$ Grâce à cette numérotation particulière aux petits motets, un chanteur aurait ainsi pu trouver aisément dans le fonds musical un répertoire d'œuvres pour sa tessiture. En réalité cette règle n'est restée que purement théorique : le manuscrit de l'In convertendo de Valette de Montigny, cité comme exemple plus haut, ne porte d'ailleurs même pas la lettre E sur sa couverture ${ }^{12}$. Pour la majorité des motets, la présentation en recueil a sûrement compliqué ce système de repérage. Pour la musique vocale profane, une mention particulière du règlement précise que l'on devra conserver un espace à la fin des livres de cantates, afin d'y placer les parties séparées qui en seront tirées, chacune dûment numérotée pour le rangement. Enfin, la dernière considération est propre aux ordres $\mathrm{A}$ et $\mathrm{B}$ : «Les pièces de musique françaises et latines à grand chœur seront rangées dans les armoires suivant l'ordre qu'elles auront été acquises et l'on trouve

\footnotetext{
${ }^{10}$ Fonctionnement de la bibliothèque, manuscrit, s.d., Bibliothèque municipale de Lyon, Rés. 134008.

${ }^{11}$ Ibid.

${ }^{12}$ François-Joseph Valette de Montigny, In convertendo, manuscrit, Bibliothèque municipale, Rés. FM 27286.
} 
l'ordre alphabétique dans une feuille attachée au-dedans de chaque armoire ». Dans cet article se trouve énoncé un élément extrêmement important, postulat préalable à toute étude du fonds musical : l'attribution de numéros aux motets à grand chœur et aux pièces lyriques françaises serait chronologique ${ }^{13}$.

Le fonctionnement de la bibliothèque requiert en principe trois inventaires pour chaque ordre : un premier, de grand format, destiné à la bibliothèque et deux annexes signées des officiers de l'académie. L'une de celles-ci devra être affectée à la bibliothèque elle-même, l'autre sera conservée dans les archives de l'académie. Le dernier paragraphe du règlement fournit des détails sur ces catalogues : «Sur le grand livre d'inventaire, il faudra laisser du papier blanc à la fin de chaque ordre, pour y mettre les pièces qui pourront venir dans la suite du même genre, et même laisser de l'espace à chaque article pour y ajouter les parties qu'on pourrait augmenter dans la suite. Ce grand livre ne pourra pas être signé, mais il y en aura deux autres semblables portés par les statuts, dont l'un restera aussi dans la bibliothèque et l'autre déposé dans les archives, où tout sera écrit de suite et vérification sera signée par les officiers à la fin de ce qui sera écrit ; après cet arrêté on écrira les nouvelles acquisitions de l'année suivante, après lequel on fera un nouvel arrêté après avoir examiné si chaque article nouveau aura été placé dans le grand livre d'inventaire dans son ordre ; ainsi des autres années. ${ }^{14}{ }$ Cette règle a-t-elle été appliquée ? Il y a un siècle, Vallas émettait déjà des doutes quant à l'existence de ces inventaires, dont les statuts de 1724 font pourtant allusion eux-aussi : «L'article VII signale l'existence de registres tenus en double. Il est possible que ce règlement soit resté lettre morte ; en tout cas, nous n'en avons trouvé aucun. (Léon Vallas, ouvr. cité, p. 58) ». En effet, les catalogues conservés ne semblent pas concorder exactement avec la description ci-dessus. Devraient en effet exister un inventaire de grand format non signé et deux autres listes, de même facture, signées de tous les officiers et du directeur de l'académie, ce qui n'est pas le cas. La feuille du règlement n'est d'ailleurs pas datée : a-t-elle été écrite dès la fondation de l'académie en 1713, en même temps que les statuts de 1724 ou plus tard? Lors de la rédaction de cette source, les inventaires mentionnés n'existent pas encore, puisque toutes les indications sont données pour leur présentation. Ce premier règlement daterait donc des débuts de l'académie ${ }^{15}$.

\footnotetext{
${ }^{13}$ Nous renvoyons à notre thèse, qui s'attache à démontrer cette chronologie effective, notamment à travers l'étude des reliures des sources (ouvr. cité, vol. I, p. 189-206).

${ }^{14}$ Fonctionnement de la bibliothèque, ouvr. cité.

${ }^{15}$ L'écriture est cependant la même que celle du catalogue de l'ordre $\mathrm{T}$ et de celui commun aux ordres $\mathrm{C}, \mathrm{M}$ et O, décrits plus loin, tous deux datés de 1748 .
} 
Les directives municipales sont toutefois très explicites, dès lors que la salle du Concert est rachetée en 1741 par le Consulat : toute la collection de musique, existant à cette date et acquise par la suite, appartient désormais à la ville et le bibliothécaire doit en fournir un catalogue $^{16}$. L'inventaire général doit être établi en double exemplaire, le premier pour le Concert, le second, déposé au bureau du secrétariat de la ville, pour le consulat. Une liste complémentaire de la musique nouvellement copiée ou achetée pendant l'année doit être remise chaque mois de janvier au secrétariat de la ville par les officiers de l'académie. Il serait logique que ces listes de mises à jour soient reportées directement sur le registre municipal. On relève, dans ces directives datant du 30 décembre 1741, une légère différence avec le descriptif du fonctionnement de la bibliothèque cité plus haut. Ici, ce ne sont pas trois mais deux catalogues qui sont mentionnés : un premier inventaire général, probablement non signé, et un second pour le consulat, signé de tous les officiers et du directeur du Concert. Certains de ces catalogues manuscrits ont été conservés. Ce sont ces différents exemplaires qui vont nous permettre de comprendre un peu mieux l'histoire de la bibliothèque. Voici donc la liste des documents conservés :

- $\quad$ Archives municipales de Lyon (dossier 0003 GG 156)

Musique latine a grand chœur sous l'ordre A, qui est dans la bibliotheque du concert de l'academie des beaux arts de la ville de Lion, 27 mai 1754 (ajouts après le 27 mai 1754 et après le 9 juin 1766), pièce 14.

Inventaire des pieces de musique françoises \& italiennes a grand cour, ordre $B$, du concert de l'academie des beaux arts, 27 mai 1754 (ajouts après le 27 mai 1754 et après le 9 juin 1766), pièce 15 .

Inventaire des pieces de musique latines, françoises et italiennes a une, deux, trois voix simphonies en concerto, en duo et trio, qui sont dans la bibliotheque du concert de l'academie des beaux arts sous les ordres suivants C. D. E. F. G. H. I. K. L. M. N. O. P. Q. R. S. T, 27 mai 1754 (ajouts en 1756 et 1764), pièce 16.

- $\quad$ Bibliothèque municipale de Lyon (dossier Rés. FM 134 008)

Inventaire des pieces de musique françoises et italiennes a grand chœur, ordre B, 1742 et 1743 (ajouts jusqu'en 1767).

Ordres C. M. O. De la bibliotheque de musique de l'académie des beaux arts, 1748.

[Inventaire des ordres $C, D, E, F, G, H, I, K, L, M, N, O, P, Q, R$ et $S$ ], 1742.

Suitte de l'ordre T, s.d.

Les ordres $\mathrm{A}$ et $\mathrm{B}$, tous les deux numérotés, font l'objet d'un inventaire séparé, probablement à cause de leur importance, l'ordre B figurant même en deux exemplaires (archives et bibliothèque). Un fragment de l'ordre T se trouve pareillement isolé. Le catalogue est commun pour les ordres $\mathrm{C}, \mathrm{M}$ et $\mathrm{O}$, qui figurent également dans un même inventaire

\footnotetext{
${ }^{16}$ Délibération consulaire, manuscrit, 30 décembre 1741, Archives municipales de Lyon, BB 306, fo 189.
} 
général des ordres $\mathrm{C}$ à $\mathrm{S}$ (ou T). Les doublons concernent donc le catalogue de l'ordre $\mathrm{B}$ d'une part, les deux catalogues des ordres C-S (ou T), d'autre part.

Les trois inventaires conservés aux archives de la ville de Lyon seraient les exemplaires fournis par le Concert au consulat. Plusieurs arguments étayent cette thèse. Remarquons tout d'abord la date commune de ces trois documents : ils ont été établis le 27 mai 1754, puis complétés par des révisions et ajouts plus tardifs. Cette date suit de près l'intronisation par le consulat d'un garde pour la bibliothèque de l'académie, qui s'est considérablement agrandie, en la personne de Coignet $^{17}$. Celui-ci, placé sous les ordres du bibliothécaire perpétuel Bergiron du Fort-Michon - et conformément à la délibération de 1741, doit établir deux inventaires généraux et pourvoir aux mises à jour annuelles, sous peine de révocation. Coignet, nommé le 2 avril, semble s'être mis immédiatement à la tâche, si l'on en juge par la proximité des dates et par l'identification de son écriture sur les inventaires des archives lyonnaises, identification que permet une comparaison de cette écriture avec sa signature apposée après les mises à jour consécutives. Les inventaires constitués devaient ensuite être visés de manière officielle par le directeur et tous les officiers du Concert. Les émargements sont précédés d'une mention explicite : «Le présent inventaire arrêté et remis au secrétariat de la ville par nous soussignés directeur et officiers de l'académie des beaux-arts, à la forme de la délibération consulaire du deux avril dernier. À Lyon le vingt-sept mai mille sept cent cinquante-quatre ». Venant confirmer le fait qu'il s'agit des exemplaires destinés à l'administration municipale, une plume du $18^{\mathrm{e}}$ siècle a ajouté dans l'angle supérieur de chaque inventaire la mention «Hôtel de ville». Enfin, les trois sources des archives municipales présentent un état complet de la collection (ordres A, B, puis $\mathrm{C}-\mathrm{T}$ ). Ils ont strictement la même présentation, les mêmes dimensions et le même papier ${ }^{18}$. Les mises à jour sont pourtant très rares et sont, le cas échéant, introduites par un petit paragraphe daté.

Les deux premiers catalogues du consulat (ordres A et B) ont subi exactement les mêmes modifications. Deux séries d'ajouts ont été faites, l'une entre 1754 et 1766, l'autre entre 1766 et 1773 environ, date de la dissolution de l'académie. Le troisième catalogue (ordre C-T) ne comporte en tout, après 1754, que deux numéros supplémentaires pour dix-sept ordres ; ces acquisitions datent précisément des années 1756 et 1764 . Les mises à jour annuelles édictées

\footnotetext{
${ }^{17}$ Délibération consulaire, manuscrit, 2 avril 1754, Archives municipales de Lyon, BB 321, fo 64 r. -65 v.

${ }^{18} \mathrm{La}$ couverture est cartonnée, de format $230 \times 365 \mathrm{~mm}$. Le cachet de l'académie figure sous le titre de chaque inventaire. Une page de garde est laissée vierge. La fin des cahiers présente aussi des folios vierges, mais dont les marges ont été tracées au crayon de bois (19 vierges pour 8 utilisés dans l'ordre A, 16 pour 5 dans l'ordre B, 8 pour 9 dans les ordres $\mathrm{C}-\mathrm{S}$ ). Le filigrane, datant de 1742, est à peu près illisible. En revanche, la contremarque représente des armoiries avec une trompe de chasse, ornée d'un ruban formant une boucle.
} 
par le consulat dans le règlement officiel n'ont donc pas été vraiment respectées. Il n'y eut en effet que deux révisions, la première s'étant opérée douze ans après l'établissement des inventaires. Les partitions ajoutées au catalogue $\mathrm{C}-\mathrm{T}$, quant à elles, semblent avoir été inscrites au moment de leur acquisition et non lors d'une mise à jour globale, comme cela a été fait pour les ordres A et B. De fait, les dates de 1756 et 1764 sont inscrites à côté du titre des œuvres musicales, sans qu'il soit spécifié, comme dans les deux premiers catalogues, qu'il s'agit de la liste des partitions entrées dans la bibliothèque à partir d'une certaine date.

Les différents catalogues conservés à la bibliothèque municipale - provenant des archives de l'académie des beaux-arts ${ }^{19}$ - sont en revanche plus difficiles à analyser. Ils sont réunis sous une reliure moderne, à côté du règlement de la bibliothèque et de la liste des différents ordres du catalogue cités plus haut. Les catalogues des ordres B et C-S sont d'une même main. Le premier des inventaires, recensant les pièces de musique française et italienne à grand chœur (ordre B), est bien plus détaillé que la source des archives municipales. Chaque numéro comporte plusieurs lignes : la première indiquant le titre et le genre, la deuxième le compositeur. Vient ensuite le détail des exemplaires s'il en existe plusieurs : il est spécifié s'il s'agit d'une partition manuscrite ou imprimée, l'édition ou l'arrangement le cas échéant ; le Concert pouvait posséder en effet jusqu'à trois versions d'une même œuvre. Un espace est laissé libre entre chaque numéro, pour l'acquisition de nouvelles versions. Le numéro de catalogue est indiqué dans la marge de gauche. En fin de ligne, quatre colonnes signalent pour chaque œuvre le nombre de parties séparées, respectivement pour les récitants (« rôles »), les choristes («voix »), l'orchestre (« instr.») et le total. Si quelques partitions n'ont pas de matériel correspondant, nombreuses sont celles dont l'effectif a été renforcé a posteriori, par l'ajout de parties supplémentaires : celles-ci sont indiquées au-dessous du jeu initial, avec le même détail accompagné de la date de l'ajout ${ }^{20}$. Ce catalogue de l'ordre B présente donc un relevé extrêmement précis, qui permettrait de savoir, à travers l'ajout de parties séparées ou des diverses annotations, si une œuvre a été jouée, dans quel effectif, si elle a été reprise, à quelle date et sous quelle forme.

\footnotetext{
${ }^{19}$ L'académie des sciences, belles-lettres et arts de Lyon a fait don du fonds musical de l'ancien Concert à la bibliothèque de la ville de Lyon, ne conservant que la correspondance académique et les écrits sur la musique.

${ }^{20}$ Un total de l'ensemble des parties est alors établi en-dessous pour chaque colonne. Le catalogue présente aussi quelques annotations, renvoyant à des cas particuliers. On peut ainsi lire, pour Les Fêtes vénitiennes de Campra $\left(n^{\circ} 1\right)$, dont le matériel a été augmenté en 1730 et $1740:$ : Les parties tirées contiennent le prologue, l'opéra, Les Devins, L'Amour saltimbanque \& Le Bal ». Les « concerts » ou extraits sont également indiqués pour les œuvres réarrangées.
} 
Le catalogue de l'ordre B comprend cent cinquante-cinq numéros. Jusqu'au $\mathrm{n}^{0} 109$, les quelques dates écrites vont de 1729 à 1741. Elles n'indiquent manifestement pas l'entrée des œuvres dans la bibliothèque, mais l'ajout de parties séparées au matériel existant ou encore l'acquisition d'une nouvelle partition. Après le $n^{0} 109$, Bergiron du Fort-Michon écrit, sans signer : «Je soussigné en qualité de bibliothécaire perpétuel du Concert reconnais que les livres et papiers de musique énoncés dans l'inventaire ci-dessus, jusques à la fin de l'année 1741, conforme à l'original de la bibliothèque, sont dans les armoires dudit concert, \& que j'en suis chargé conjointement avec M. Mayeuvre inspecteur du Concert à Lyon Ce $[s i c]$ ». La mention « conforme à l'original » indiquerait que cet inventaire n'est pas celui qui a servi de modèle, mais qu'il existe en plus une source de référence. C'est probablement le troisième catalogue que signalait le règlement de la bibliothèque et qui n'était pas mentionné dans le texte officiel du 30 décembre 1741. Cette source originale, aujourd'hui perdue, était peut-être destinée à la bibliothèque elle-même ; l'exemplaire conservé à la bibliothèque municipale devait être celui des archives de l'académie. La graphie initiale change pour les $\mathrm{n}^{\text {os }} 110-155$. Ceux-ci comportent presque tous une date comprise entre 1741 et 1767 . Les œuvres sont classées de façon chronologique, les dernières n'étant pas datées. À partir du $\mathrm{n}^{\mathrm{o}} 122$, on reconnaît l'écriture de Coignet, le garde de la bibliothèque. Ce catalogue de l'ordre $\mathrm{B}$ a donc été commencé dès la fondation de l'académie, en 1713. Les quatre-vingt-un premiers numéros datent d'avant 1729, puisqu'à partir de cette année on commence à ajouter des parties séparées supplémentaires aux opéras et ballets déjà inventoriés. La liste est mise à jour en 1742, date à laquelle Bergiron la vérifie et la certifie ; Coignet va faire des ajouts jusqu'en 1767, année de la dernière œuvre recensée. Après 1750, l'écriture de Bergiron disparaît et Coignet est le seul à noter les nouvelles acquisitions. Cette source, la seule qui fut mise régulièrement à jour, correspond donc à l'inventaire officiel du Concert établi après le rachat des biens de l'académie par la ville de Lyon en 1741. Les dates de 1742 et 1743, inscrites sur la couverture, le confirment.

À la bibliothèque municipale de Lyon, les ordres $\mathrm{C}$ à $\mathrm{S}$ sont rassemblés dans un même cahier. L'histoire de cet inventaire est la même que celle de l'ordre B. L'écriture est semblable, mais la présentation est différente : les œuvres sont listées les unes après les autres, sans détail. On y retrouve le même paragraphe de Bergiron attestant de la musique conservée à la bibliothèque à la fin de l'année 1741. L'inventaire date donc vraisemblablement du début de l'année 1742. Coignet y a fait de rares corrections ou renvois par la suite, mais aucun ajout. 
Il n'y a d'ailleurs pas de place pour cela dans la mise en page, contrairement au catalogue précédent.

De l'inventaire de l'ordre T n'est conservée que la dernière page, « suite de l'ordre $T$ ». En réalité, il est possible qu'il s'agisse de la totalité de l'ordre $\mathrm{T}$, et non pas d'un fragment ${ }^{21}$. Ce n'est ni Coignet, ni le copiste des inventaires des ordres $\mathrm{B}$ et $\mathrm{C}-\mathrm{S}$, mais une troisième main qui dresse cette liste. La graphie est la même que celle du catalogue des ordres $\mathrm{C}, \mathrm{M}$ et $\mathrm{O}$ de 1748, mais aussi que celle du règlement de la bibliothèque et de la description des ordres des pièces de musique, mentionnés plus haut. Tous ces documents sont d'ailleurs reliés ensemble, sous la cote Rés. FM 134008.

Le catalogue de la bibliothèque municipale rassemblant les ordres $\mathrm{C}, \mathrm{M}$ et $\mathrm{O}$ est très particulier, et ce pour plusieurs raisons. La graphie, d'abord, si elle est identique à celle de l'ordre $\mathrm{T}$, est complètement inconnue ${ }^{22}$. Bergiron a ajouté l'ex-libris « De la bibliothèque de musique de l'académie des beaux-arts, $1748 \gg$ sur la couverture. Pourtant, la copie n'est ni de lui, ni de la main de Coignet, qui joint seulement un dernier numéro à l'ordre O. L'inventaire sélectionne toute la musique instrumentale de la bibliothèque (si l'on excepte les pièces pour trompette marine, classées dans l'ordre T): symphonies à grand concert (ordre C) et symphonies en duo et trio (ordre O). Il pourrait donc s'agir d'un répertoire à l'usage des symphonistes. Mais l'inventaire contient aussi les partitions des seules cantates françaises (ordre M), ce qui est difficilement explicable, car les petits motets ou les cantates italiennes pourraient tout aussi bien y figurer. De plus, les ordres $\mathrm{C}$ et $\mathrm{O}$ font état de musique en parties séparées, alors que l'ordre $\mathrm{M}$ ne contient que des partitions. Le catalogue est le seul à ne pas être signé et à ne donner aucune information quant à son auteur, et la date de 1748 ne semble pas constituer un indice.

La comparaison des différents inventaires, conservés à la bibliothèque et aux archives de Lyon, se révèle intéressante à plusieurs titres; elle permet notamment d'apporter des précisions sur ce catalogue particulier des ordres C, M et $\mathrm{O}$ de 1748 et d'en justifier la présence. Si l'on confronte les catalogues du consulat (1754, archives) à ceux de l'académie des beaux-arts (1742, bibliothèque), il ressort, par exemple, que l'ordre A n'a été conservé

\footnotetext{
${ }^{21}$ La comparaison avec l'ordre $\mathrm{T}$ du catalogue du consulat conservé aux archives municipales, absolument identique, montre qu'il n'existe aucun titre précédant ceux qui sont indiqués dans cette « suite de l'ordre T ».

${ }^{22}$ Nous émettons une réserve : il pourrait éventuellement s'agir de la même écriture que celle qui a copié les ordres $\mathrm{B}$ et $\mathrm{C}-\mathrm{S}$ conservés à la bibliothèque municipale, mais la graphie de cet inventaire est trop négligée, par rapport à celles des autres documents très bien calligraphiés, pour permettre une comparaison.
} 
qu'aux archives municipales, cependant que les ordres $\mathrm{B}$ et $\mathrm{C}-\mathrm{T}$ sont en double exemplaire. L'inventaire de l'ordre B du Concert est bien plus détaillé que la copie remise au consulat, laquelle recense uniquement le total des parties séparées, ne tenant pas compte du détail des augmentations successives. En revanche, les listes des opus sont strictement identiques et comportent le même nombre de numéros, puisque l'exemplaire de 1742 a été complété au fur et à mesure. Seuls les $\mathrm{n}^{\text {os }} 120-121$ et $127-128$ sont intervertis dans la copie ${ }^{23}$. Le catalogue de 1754 ne présente pas les différentes étapes d'acquisitions signalées dans celui de 1742. Les deux sources du catalogue $\mathrm{C}-\mathrm{S}$ (ou T) détaillent de la même façon les volumes de musique. Mais l'exemplaire du consulat, plus tardif, comporte plus de numéros, ce qui permet d'identifier les partitions ou parties séparées qui sont rentrées dans la collection du Concert après 1742. Il englobe aussi l'ordre T, qui se trouve en exemplaire séparé dans les archives de l'académie. L'ordre C ne comporte pas moins de dix-huit numéros supplémentaires dans le catalogue du consulat (archives, 1742), alors que seulement dix-neuf étaient répertoriés au total en 1742 dans le catalogue du Concert (bibliothèque, 1754). L'académie a donc doublé son répertoire de symphonies en douze ans. Les deux premières œuvres n'ont pas le même nombre de parties séparées sur l'inventaire de la ville et sur celui de l'académie ${ }^{24}$. Par la suite, Coignet ajoute une correction à propos du concerto de Corelli, premier numéro de l'ordre $\mathrm{C}$, correction visible sur les deux catalogues de 1742 et 1748 (bibliothèque municipale), tandis que le texte a été directement modifié sur celui de 1754 (archives municipales) ${ }^{25}$. Ce détail certifie les datations des inventaires, le plus tardif ayant intégré les corrections. Quatre livres de cantates ont rejoint l'ordre $M$ après 1742 : l'inventaire du consulat comporte onze numéros au lieu de sept pour la source du Concert. L'ordre O est assez différent dans les deux inventaires. Globalement, sur la source consulaire, il n'y a qu'une vingtaine de titres en 1742 , contre cinquante et un numéros en 1754. Mais il n'est pas aisé d'établir une correspondance entre les deux listes, tant les œuvres - volumes de sonates ou de duos, gravés ou manuscrits sont mélangées. Les inventaires des autres ordres (D, E, F, G, H, I, K, L, N, P, Q, R, S et T) sont absolument conformes. Le texte détaillé qui introduit l'ordre $\mathrm{T}$ est strictement le même dans les deux sources ; la mention du dernier titre, oublié et ajouté en bas de liste, a été copiée

\footnotetext{
${ }^{23}$ «Zä̈s, Ballet héroïque par $\mathrm{M}^{\mathrm{r}}$ Rameau » porte le numéro 120 dans l'inventaire de l'académie, 121 dans celui du consulat; c'est le contraire pour le « Ballet des Grâces par $\mathrm{M}^{\mathrm{r}}$ Mouret». «Zélindor divertissement par $\mathrm{M}^{\mathrm{rs}}$ Rebel et Francœur porte le numéro 127 dans l'inventaire de l'académie, 128 dans celui du consulat ; il est interverti avec «La Guirlande ballet par $\mathrm{M}^{\mathrm{r}}$ Rameau ».

${ }^{24}$ Ceci ne peut faire penser à une augmentation du matériel entre 1742 et 1754 , car si pour le $n^{\circ} 2$ le catalogue de 1754 annonce un nombre supérieur de parties séparées, c'est l'inverse pour le $\mathrm{n}^{\circ} 1$.

${ }^{25} \mathrm{Au} \mathrm{n}^{\mathrm{o}} 1$ de l'ordre $\mathrm{C}$ (Bibliothèque municipale, catalogues $\mathrm{C}-\mathrm{S}, 1742 ; \mathrm{C}, \mathrm{M}$ et $\mathrm{O}, 1748$ ), on peut lire : «nota : que ce $1^{\text {er }}$ article n'est que de huit parties, les 8 autres ont été troquées avec $\mathbf{M}^{\mathrm{r}}$ Delatourette pour des motets de Bernier ».
} 
à l'identique. Il n'y a donc que les ordres C, M et $\mathrm{O}$ qui aient été augmentés entre 1742 et 1754. Ces ajouts auraient dû être reportés sur l'inventaire du Concert. Or le catalogue conservé dans la bibliothèque de l'académie n'a pas été mis à jour. En effet, au lieu de compléter les inventaires de 1742, les académiciens ont préféré créer de nouveaux catalogues propres aux ordres $\mathrm{C}, \mathrm{M}$ et $\mathrm{O}$, dont la liste s'était agrandie. Ce sont ces annexes qui sont conservées aujourd'hui. Elles contiennent trente-sept numéros pour l'ordre $\mathrm{C}$, onze pour l'ordre $\mathrm{M}$ et cinquante et un pour l'ordre $\mathrm{O}$. La liste de cette dernière partie correspond exactement à celle du catalogue de 1754, donné au consulat. Elle constitue, par conséquent, une mise à jour du premier inventaire devenu caduque.

Les différents catalogues conservés correspondent donc bien à l'application des règlements édictés par la ville de Lyon. Le premier date de 1742, juste après que le consulat a racheté l'hôtel $\mathrm{du}$ Concert et sa collection de musique. Le bibliothécaire fait alors copier des inventaires pour l'académie, inventaires conservés aujourd'hui à la bibliothèque municipale. Mais leur mise en page ne permet pas d'ajouter les œuvres nouvellement acquises. C'est pourquoi en 1748 il devient nécessaire de faire un nouvel inventaire pour les ordres $\mathrm{C}, \mathrm{M}$ et $\mathrm{O}$ qui ont été considérablement augmentés. La similitude d'écriture entre le règlement de la bibliothèque et la nomenclature des ordres cités plus haut pourrait indiquer qu'ils ont été établis par une même personne en 1748. En 1754, lorsque Coignet est institué garde de la bibliothèque par le consulat, il doit établir à nouveau des inventaires de la musique du Concert. Ce sont les manuscrits conservés aujourd'hui aux archives municipales.

Mais les informations que livrent les catalogues de la collection constituent-elles une source exacte? La seule indication pour le musicologue se trouve dans la correspondance entre les inventaires du $18^{\mathrm{e}}$ siècle et les sources musicales conservées aujourd'hui dans le fonds de l'académie. Cependant, pour les partitions et matériels disparus, nous ne pouvons que nous référer au catalogue manuscrit de l'académie. Or, celui-ci présente quelques singularités. La première concerne le classement lui-même. L'ordre A du catalogue ne contient pas uniquement des motets français à grand chœur et symphonie, contrairement à ce qu'indique pourtant son intitulé. Ainsi pourrait être discutée la présence de messes en musique et de certains motets italiens. Pourtant ces deux derniers genres adoptent toujours le latin - excepté certaines pièces de la messe - et un effectif identique à celui du Grand motet, à savoir des solistes, un chœur et un orchestre. Leur place dans l'ordre A se justifie donc par la 
langue utilisée et l'effectif. En revanche, les motets Buccince litui classica de Scarlatti $\left(\mathrm{n}^{\circ} 118\right)$ et Qui habitat de Stradella ( $\left.\mathrm{n}^{\circ} 130\right)$, légués par Antoine Hedelin au Concert, posent un problème différent ${ }^{26}$. Ils sont tous deux indexés dans l'ordre $\mathrm{A}$, bien que leur effectif ne justifie pas ce classement. Le motet de Scarlatti est « un motet à cinq voix, deux sopranos, alto, ténor et basse, avec violons et trompette, et remplissage », pour lequel le catalogue mentionne onze parties séparées, dont six parties instrumentales sont conservées ${ }^{27}$. Les cinq parties perdues correspondent donc nécessairement aux cinq voix, ce qui porte l'effectif à cinq solistes accompagnés d'un ensemble instrumental. Il s'agirait donc d'un motet à voix seule, sans chœur. Cette hypothèse est confortée par le fait qu'il s'agit d'une œuvre relativement courte ( 5 sections, pour un total de 217 mesures) et que, si les parties séparées comportent l'incipit de chaque verset, ne s'y trouve aucune indication d'effectif pour chaque mouvement - du type «chœur » ou «duo»-, comme c'est généralement le cas pour une œuvre chorale. Quant à la composition de Stradella, il s'agit aussi d'une pièce à cinq voix seules, soutenue par une basse-continue, sans symphonie. Ces deux motets auraient donc dû être rangés et inventoriés dans d'autres ordres de la bibliothèque. Cependant, parmi ceux-ci, aucun n'inclut des œuvres à cinq voix. Tout au plus l'ordre L rassemble-t-il les « partitions de motets à une, deux, trois, quatre voix avec et sans symphonies ». Les deux compositions italiennes ont donc été placées par défaut avec les motets à grand chœur et symphonie, alors qu'elles n'appartiennent pas à ce genre musical français. Cette anomalie dans le classement a pu être identifiée sur ces deux sources conservées, mais d'autres pièces, aujourd'hui perdues, ont peut-être été également rangées dans un ordre auquel leur effectif vocal ou instrumental ne les destinait pas.

Si l'acquisition d'une version différente d'une même œuvre apparaît très clairement dans le catalogue de l'ordre $\mathrm{B}$ conservé à la bibliothèque municipale, ce n'est pas le cas dans les autres sources. L'inventaire de l'ordre A se répète ainsi parfois, répertoriant le même motet sous des numéros différents, comme, par exemple, l'In convertendo de Jean-Philippe Rameau - n77 (partition et 60 parties séparées) et n²19 (partition seule). Le premier numéro correspond sûrement à l'œuvre telle quelle a été composée à Lyon au début du $18^{\mathrm{e}}$ siècle, acquise par l'académie à sa fondation ou peu après que Rameau eut quitté Lyon, en 1715. La deuxième partition est probablement une version plus tardive de l'œuvre, remaniée, telle

\footnotetext{
26 Alessandro Scarlatti, Buccince litui, manuscrit, Bibliothèque municipale de Lyon, Rés. FM 133950 ; Alessandro Stradella, Qui habitat, manuscrit, Bibliothèque municipale de Lyon, Rés. FM 133936.

27 «Motteto a cinque voci [...] due soprani alto tenore e basso con violini e tromba e ripieni ». Les parties séparées conservées sont les suivantes: «organo», «basso continuo», «violino ripieni », « tromba con violino », «basse de symphonie » et « violino solo».
} 
qu'elle a été entendue au Concert spirituel pour la première fois le 30 mars 1751. L'académie l'aurait fait copier après son exécution parisienne. L'In convertendo de Rameau apparaît sous deux numéros différents et constitue un cas spécifique. Généralement, les inventaires ne font pas état des doublons, partitions acquises, offertes ou léguées alors que le Concert possédait déjà une source de l'œuvre. Ces exemplaires supplémentaires sont usuellement inventoriés sous le numéro attribué au premier volume. Le cas le plus significatif est celui des vingt et un livres de motets de Lalande, gravés en 1729, motets dont l'académie possédait déjà plusieurs copies manuscrites, souvent dans une première version ${ }^{28}$. Lorsque les éditions arrivent dans la bibliothèque, Bergiron range les œuvres gravées dont le Concert possède déjà un volume avec celui-ci et catalogue les autres sous des nouveaux numéros $\left(\mathrm{n}^{\text {os }} 173-207\right)^{29}$. Les premiers numéros des motets de Lalande correspondent donc à deux partitions : une copie manuscrite et un exemplaire gravé. Les sources conservées sont même au nombre de trois pour le Notus in Judcea Deus $\left(\mathrm{n}^{\mathrm{o}}\right.$ 177) : deux partitions manuscrites, une gravée ${ }^{30}$. Si quarante-trois compositions du sous-intendant de la Chapelle royale sont donc répertoriées dans l'ordre A, la bibliothèque de l'académie devait en réalité en posséder bien plus. Or ceci n'apparaît aucunement à la lecture du catalogue.

Certaines œuvres appartenant à la collection n'auraient pas été inventoriées. Ainsi, la partition du Beati omnes qui timent de Campra à grand chœur, pourtant estampillée du cachet de l'académie des beaux-arts, ne figure pas au catalogue du Concert. Elle possède même un numéro de classement écrit en haut de la reliure $\left(\mathrm{n}^{\circ} 280\right)$, auquel une deuxième main a ajouté par la suite l'annotation « ou $\mathrm{n}^{\mathrm{o}} 272 »$. Cependant, dans l'inventaire de l'ordre A, le $\mathrm{n}^{\mathrm{o}} 272$ correspond au Deus venerunt gentes de Madin, tandis que le $\mathrm{n}^{0} 280$ est un Confitemini de Campra. Non seulement les numéros ne concordent donc pas avec la partition, mais encore sur les dix-huit motets de Campra recensés dans le catalogue, aucun n'a pour titre Beati omnes. Les cas similaires sont trop nombreux pour être détaillés dans les limites de cet article ; ils concernent les partitions aussi bien que le total des parties séparées qui sont parfois conservées, tandis qu'elles n'étaient pas mentionnées dans l'inventaire. Les Affiches de Lyon

\footnotetext{
${ }^{28}$ Quarante grands motets de Lalande en partition réduite seront gravés après sa mort, en 1729, par Louis-Hector Hüe (RISM L 303-322).

${ }^{29}$ Dans cette série s'intercalent l'In exitu Israël de Campra ( ${ }^{\circ}$ 178) et le Te Deum de Colin de Blamont gravé en $1732\left(n^{\circ} 183\right)$. Le n ${ }^{\circ} 187$ n'est pas attribué.

30 La bibliothèque municipale de Lyon conserve une partition gravée (Rés. FM 27 325) et manuscrite (Rés. FM 27 308). La bibliothèque de Reims, dont le fonds patrimonial avait été fortement endommagé à la suite du premier conflit mondial, s'est vue offrir le deuxième manuscrit lyonnais (Bibliothèque Carnegie, Ms 2 840). Les deux copies sont de la même main et ne présentent que d'infimes différences.
} 
signalent ainsi à plusieurs reprises qu'une œuvre a été jouée au concert, sans que l'académie semble en avoir possédé la partition.

Enfin, si les œuvres ont été classées suivant l'ordre chronologique de leur arrivée dans la bibliothèque, le catalogage a de toute évidence été fait par séries. Le bibliothécaire procédait au rangement et au numérotage lorsqu'il avait quelques volumes de musique récemment arrivés ou qu'il était en retard dans son travail. C'est pourquoi, dans l'ordre A, par exemple, les motets de Salomon ont été inventoriés ensemble : le De profundis ( $\mathrm{n}^{\circ}$ 66, Rés. FM 28 325) porte pourtant la mention «deuxième motet », l'In te Domine speravi (nº 67, Rés. FM 28 327) devant être le premier. De la même façon, l'Exurgat Deus de Lalande, donné par M. Poulletier de Nainville en 1725 (nº 149, Rés. FM 133 630) est classé avant le Cantate Domino du même auteur, donné par sa femme l'année précédente $\left(\mathrm{n}^{\mathrm{o}} 150\right.$, Rés. FM 133 657) ${ }^{31}$. Une chronologie inversée apparaît également en comparant les datations du Credidi propter de Belissen ( $n^{\circ} 245$, non conservé), fourni par Bergiron en août 1761, et du Dominus regnavit de Belouard (n 249, Rés. FM 129 928), dont les parties séparées datent de mai $1760^{32}$. Le bibliothécaire n'effectuait peut-être sa mise à jour qu'une fois l'an. L'ordre chronologique n'est donc pas fiable dans le détail.

Les différents catalogues, s'ils restent un outil de référence précis et indispensable, doivent être utilisés avec précaution. Les questions de conformité d'effectif, de doublons, d'œuvres non répertoriées ou encore de chronologie indiquent des spécificités de l'histoire de la collection, que la liste continue des volumes de musique ne fait pas apparaître. Malgré tout, la présence des inventaires manuscrits reste l'une des plus grandes richesses du fonds musical de l'académie des beaux-arts. Elle apporte autant d'indices sur la constitution d'une bibliothèque musicale au $18^{\mathrm{e}}$ siècle, que sur le répertoire lyonnais, l'histoire des partitions, leur interprétation et leur exécution. La connaissance des catalogues ne constitue évidemment qu'un point de départ pour le musicologue. Si les inventaires offrent une vue d'ensemble du corpus du Concert de Lyon et constituent la mémoire de sa bibliothèque, il convient de les confronter directement aux sources musicales conservées, démarche préalable à l'observation et à l'investigation de l'ensemble du fonds lyonnais.

\footnotetext{
${ }^{31}$ Les dons de l'intendant de Lyon et de sa femme sont rappelés par une pièce de titre sur chaque couverture, précisant la date.

${ }^{32}$ Ces dates sont certifiées par deux pièces de comptabilité : Comptabilité municipale, $1^{\text {er }}$ mai 1760 et 27-28 août 1761, manuscrit, archives municipales de Lyon, CC 3408, pièces 64 et 68.
} 